\title{
The Issue Flow Concave Surface Flow in Compressible Fluid
}

\author{
Samara T. Zhusupbekova* \\ Kyrgyz National Agrarian \\ University im. K.I. Skryabina
} 68 Mederova, Bishkek, 720005, Kyrgyzstan

Received 12.03.2015, received in revised form 27.07.2015, accepted 20.11.2015

The article deals with Gertler vortices in the boundary layer of fluid applied typical range of variation of the wavelength of the vortices. Formulated boundary problem for the typical shortwavelength regime. The development of vortices Gertler in compressible, especially hypersonic flows, attracted by now considerable interest in the development of new technologies, namely the creation of new supersonic little turbulent wind tunnel and the design of hypersonic aircraft, which detect the position of the laminar-turbulent transition and finding the distributions and peak values of the heat flows are key elements. In this article the mathematical model describing the nonlinear stage of development of disturbances in compressible boundary layer in the field of centrifugal forces at large, but subcritical Reynolds numbers and Gertler. This model is designed for the description of the vortices Gertler, and to describe the perturbed flows about local or periodic irregularities in the transverse direction or the other spatial disturbed flow. For small amplitudes of perturbations of nonlinear boundary value problems are reduced to linear problems of the theory of stability, which so far developed only in fragments. Based on the method of matched asymptotic expansions we studied some linear modes of perturbations in the field of centrifugal forces. In particular, The role of the transition temperature layer in the development of disturbances in hypersonic flows. At the same time, the experimental studies suggest the occurrence of other possible forms of perturbed vortex movement of gas that requires further research.

Keywords: perturbed region, Gertler Taylor vortices, the thickness of the boundary layer, transversal speed.

Citation: Zhusupbekova S.T. The issue flow concave surface flow in compressible fluid, J. Sib. Fed. Univ. Eng. technol., 2016, 9(1), 32-38. DOI: 10.17516/1999-494X-2016-9-1-32-38.

(C) Siberian Federal University. All rights reserved

* Corresponding author E-mail address: samaraj-t@mail.ru 


\title{
К вопросу обтекания вогнутой поверхности потоком сжимаемой жидкости
}

\author{
С.T. Жусупбекова \\ Кыргызский национальный аграрный \\ университет им. К.И. Скрябина \\ Кыргызстан, 720005, Бишкек, Медерова, 68
}

В статье рассматриваются вихри Гертлера в пограничном слое жидкости применительно к характерным диапазонам изменения длины волны вихрей. Формулируется краевая задача для характерного коротковолнового режима. Развитие вихрей Гертлера в сжимаемых, в частности гиперзвуковых, потоках привлекает в настоящее время значительный интерес в связи с разработкой новых технологий, а именно с созданием новых сверхзвуковых малотурбулентных аэродинамических труб и с проектированием гиперзвуковых летательных аппаратов, для которых определение положения ламинарно-турбулентного перехода и нахождение распределений и пиковых величин тепловых потоков являются ключевыми элементами. В настоящей статье сформулирована математическая модель, описывающая нелинейную фазу развития возмущений в сжимаемом пограничном слое в поле центробежных сил при больших, но докритических значениях чисел Рейнольдса и Гертлера. Эта модель предназначена для описания как развития вихрей Гертлера, так и возмущенных течений около локальных или периодических в трансверсальном направлении неровностей или других пространственных возмущенных течений. При малых амплитудах возмущений нелинейные краевые задачи сводятся к линейным задачам теории устойчивости, которая к настоящему времени разработана лишь фрагментарно. На основе метода сращиваемых асимптотических разложений исследованы некоторые линейные режимы развития возмущений в поле центробежных сил. В частности, выявлена роль переходного температурного слоя в развитии возмущений в гиперзвуковых потоках. В то же время имеющиеся экспериментальные исследования свидетельствуют о возникновении других возможных форм возмущеенного вихревого движения газа, что требует проведения дальнейших исследований.

Ключевые слова: возмущенная область, вихри Гертлера - Тейлора, толщина пограничного слоя, трансверсальная скорость.

Рассмотрим режим, соответствующий длинам волны вихрей Гертлера - Тейлора $\lambda$, сравнимым с толщиной пограничного слоя $\delta$. В этом случае характерные размеры возмущенной области течения совпадают по порядку величины, тогда одинаковые порядки будут иметь и возмущенные величины вертикальной и трансверсальной скоростей $\mathrm{v} \sim \mathrm{W}$, что следует из уравнения неразрывности и принципа минимального вырождения.

Предположим, что вихри вызывают нелинейные изменения основного течения, тогда

$$
\mathrm{u} \sim \Delta \mathrm{u} \sim 0 .
$$

Нелинейность, проявляющаяся в трансверсальном направлении, дает

$$
\mathrm{w} \sim \Delta \mathrm{w} \sim(\Delta \mathrm{p})^{\overline{2}} .
$$

При этом исследуются нелинейные процессы, проявляющиеся в течении с характерным масштабом в направлении координаты $z$, равным $\lambda$, что определяет величину градиента давления в поперечном направлении: 


$$
\frac{\partial \mathrm{p}}{\partial \mathrm{y}} \sim \frac{\rho \mathrm{u}^{2}}{\varkappa}
$$

Отсюда $\Delta \mathrm{p} \sim \varepsilon \mathcal{~}$. Далее $\mathrm{v} \sim \mathrm{w} \sim \varepsilon^{\frac{1}{2}} \mathcal{X}^{\frac{1}{2}}$.

Из уравнения неразрывности и оценок (1) - (3) можно найти продольный размер возмущенной области

$$
\mathrm{x} \sim \varepsilon^{\frac{1}{2}} \mathcal{X}^{\frac{1}{2}}
$$

Для дальнейшего анализа важно оценить порядок отношения диффузионного и инерционного членов в уравнении продольного импульса

$$
\frac{\mu \frac{\partial^{2} u}{\partial y^{2}}}{\rho u \frac{\partial u}{\partial x}} \sim 0\left(\varepsilon^{\frac{1}{2}} \mathcal{X}^{\frac{1}{2}}\right) \rightarrow 0 .
$$

Понятно, что в таких условиях не выполнено условие прилипания и поэтому необходимо ввести на дне основной области подслой с толщиной

$$
\mathrm{y} \sim \varepsilon^{\frac{3}{4}} x^{-\frac{1}{4}}
$$

течение, в котором будет описываться системой уравнений трехмерного пограничного слоя. Решение такой системы можно представить в виде

$$
\begin{aligned}
& \mathrm{x} \approx 1+\lambda \varepsilon^{-\frac{1}{2} \mathcal{X}^{-\frac{1}{2}}} \mathrm{X}_{\alpha} \\
& \mathrm{y} \approx \lambda \mathrm{y}_{\alpha} \\
& \mathrm{z} \approx \lambda \mathrm{z}_{\alpha} \\
& \mathrm{u}(\mathrm{x}, \mathrm{y}, \mathrm{z}, \varepsilon) \approx \mathrm{u}_{\alpha}\left(\mathrm{x}_{\alpha}, \mathrm{y}_{\alpha}, \mathrm{z}_{\alpha}\right)+\cdots \\
& \mathrm{v}(\mathrm{x}, \mathrm{y}, \mathrm{z}, \varepsilon) \approx \mathrm{v}_{\alpha}\left(\mathrm{x}_{\alpha}, \mathrm{y}_{\alpha}, \mathrm{z}_{\alpha}\right) \cdot \lambda^{-1} \varepsilon^{\frac{3}{2}} \mathcal{X}^{\frac{1}{2}}+\cdots \\
& \mathrm{w}(\mathrm{x}, \mathrm{y}, \mathrm{z}, \varepsilon) \approx \mathrm{w}_{\alpha}\left(\mathrm{x}_{\alpha}, \mathrm{y}_{\alpha^{\prime}}, \mathrm{z}_{\alpha}\right) \cdot \varepsilon^{\frac{1}{2}} \mathcal{\chi}^{\frac{1}{2}}+\cdots \\
& \rho(\mathrm{x}, \mathrm{y}, \mathrm{z}, \varepsilon) \approx \rho_{\alpha}\left(\mathrm{x}_{\alpha}, \mathrm{y}_{\alpha}, \mathrm{z}_{\alpha}\right)+\cdots \\
& \mathrm{H}(\mathrm{x}, \mathrm{y}, \mathrm{z}, \varepsilon) \approx \mathrm{H}_{\alpha}\left(\mathrm{x}_{\alpha}, \mathrm{y}_{\alpha}, \mathrm{z}_{\alpha}\right)+\cdots
\end{aligned}
$$

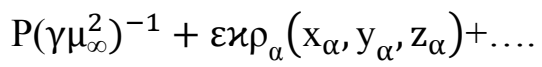

Подстановка этих разложений в систему уравнений Навье - Стокса и совершение предельного перехода при $\mathrm{Re} \rightarrow \infty, \mathrm{Ge} \rightarrow \infty$ приводят к модифицированной системе уравнений Эйлера для трехмерного возмущенного течения: 


$$
\begin{aligned}
& \frac{\partial\left(\rho_{\alpha} \mathrm{u}_{\alpha}\right)}{\partial \mathrm{x}_{\alpha}}+\frac{\partial\left(\rho_{\alpha} \mathrm{v}_{\alpha}\right)}{\partial \mathrm{y}_{\alpha}}+\frac{\partial\left(\rho_{\alpha} w_{\alpha}\right)}{\partial \mathrm{z}_{\alpha}}=0 \\
& \mathrm{u}_{\alpha} \frac{\partial \mathrm{u}_{\alpha}}{\partial \mathrm{x}_{\alpha}}+\mathrm{v}_{\alpha} \frac{\partial \mathrm{u}_{\alpha}}{\partial \mathrm{y}_{\alpha}}+w_{\alpha} \frac{\partial \mathrm{u}_{\alpha}}{\partial \mathrm{z}_{\alpha}}=0 \\
& \beta\left[\mathrm{u}_{\alpha} \frac{\partial \mathrm{v}_{\alpha}}{\partial \mathrm{x}_{\alpha}}+\mathrm{v}_{\alpha} \frac{\partial \mathrm{v}_{\alpha}}{\partial \mathrm{y}_{\alpha}}+\mathrm{w}_{\alpha} \frac{\partial \mathrm{v}_{\alpha}}{\partial \mathrm{z}_{\alpha}}\right]+\mathrm{u}_{\alpha}^{2}+\frac{1}{\rho_{\alpha}} \frac{\partial \rho_{\mathrm{x}}}{\partial \mathrm{y}_{\alpha}}=0 \\
& {\left[\mathrm{u}_{\alpha} \frac{\partial \mathrm{w}_{\alpha}}{\partial \mathrm{x}_{\alpha}}+\mathrm{v}_{\alpha} \frac{\partial \mathrm{w}_{\alpha}}{\partial \mathrm{x}_{\alpha}}+\mathrm{w}_{\alpha} \frac{\partial \mathrm{w}_{\alpha}}{\partial \mathrm{x}_{\alpha}}\right]+\frac{1}{\rho_{\alpha}} \frac{\partial \rho_{\mathrm{x}}}{\partial \mathrm{z}_{\alpha}}=0} \\
& \mathrm{u}_{\alpha} \frac{\partial \mathrm{H}_{\alpha}}{\partial \mathrm{x}_{\alpha}}+\mathrm{v}_{\alpha} \frac{\partial \mathrm{H}_{\alpha}}{\partial \mathrm{y}_{\alpha}}+\mathrm{w}_{\alpha} \frac{\partial \mathrm{H}_{\alpha}}{\partial \mathrm{z}_{\alpha}}=0 \\
& \beta=\frac{\varepsilon^{2}}{\lambda^{2}}
\end{aligned}
$$

Отличие системы уравнений (6) от обычной системы уравнений Эйлера состоит в наличии члена в уравнении для поперечного импульса, учитывающего влияние центробежных сил.

Другая особенность связана с отсутствием градиента давления в уравнении продольного импульса. Из второго уравнения системы (6) следует сохранение продольной составляющей вектора скорости вдоль линии тока. Аналогичным первым интегралом обладает и уравнение для полной энтальпии. Для возмущений малой амплитуды решение системы уравнений (6) можно представить в виде

$$
\begin{gathered}
\mathrm{u}(\mathrm{x})=\mathrm{u}_{0}\left(\mathrm{y}_{\alpha}\right)+\tau \mathrm{u}_{\alpha}\left(\mathrm{y}_{\alpha}\right) \sin \mathrm{z}_{\alpha} \exp \left(\alpha \mathrm{x}_{\alpha}\right)+\cdots \\
\mathrm{v}_{\alpha}=\tau \mathrm{v}_{\alpha}\left(\mathrm{y}_{\alpha}\right) \exp \left(\alpha \mathrm{x}_{\alpha}\right) \sin \mathrm{z}_{\alpha}+\ldots . \\
\mathrm{w}_{\alpha}=\tau \mathrm{w}_{\alpha}\left(\mathrm{y}_{\alpha}\right) \exp \left(\alpha \mathrm{x}_{\alpha}\right) \operatorname{cosz}_{\alpha}+\ldots . \\
\mathrm{H}_{\alpha}=\mathrm{H}_{0}\left(\mathrm{y}_{\alpha}\right) \tau \mathrm{G}_{0}\left(\mathrm{y}_{\alpha}\right) \exp \left(\alpha \mathrm{x}_{\alpha}\right) \operatorname{sinz}_{\alpha}+\ldots \ldots \\
\rho_{\alpha}=\mathrm{r}_{0}\left(\mathrm{y}_{\alpha}\right)+\tau \mathrm{R}_{\alpha}\left(\mathrm{y}_{\alpha}\right) \exp \left(\alpha \mathrm{x}_{\alpha}\right) \sin \mathrm{z}_{\alpha}+\ldots \ldots \\
\mathrm{P}_{\alpha}=\mathrm{P}_{0}\left(\mathrm{y}_{\alpha}\right)+\tau \mathrm{P}_{\alpha}\left(\mathrm{y}_{\alpha}\right) \exp \left(\alpha \mathrm{x}_{\alpha}\right) \operatorname{sinz} \mathrm{z}_{\alpha}+\ldots . \\
\tau \ll 1
\end{gathered}
$$

Ограничиваясь нулевым и первым приближением из (6) и (7), можно получить систему обыкновенных дифференциальных уравнений вида

$$
\begin{aligned}
& \alpha \rho_{0} u_{\alpha}+\alpha u_{\alpha} R_{\alpha}+\rho_{0} v_{\alpha}^{\prime}+v_{\alpha} \rho_{0}^{\prime}-\rho_{0} w_{\alpha}=0 \\
& \alpha u_{0} u_{\alpha}+v_{\alpha} u_{0}^{\prime}=0 \\
& \beta\left[\alpha u_{0} u_{\alpha}+2 u_{0} u_{\alpha}\right]+\frac{1}{\rho_{0}} P_{\alpha} u_{0}^{\prime}-\frac{R_{\alpha}}{\rho_{0}^{2}} \rho_{0}^{\prime}=0
\end{aligned}
$$




$$
\begin{aligned}
& \frac{1}{\rho_{0}} P_{0}^{\prime}=-u_{0}^{2} \\
& \alpha u_{0} w_{\alpha}+\frac{R_{\alpha}}{\rho_{0}}=0 \\
& \alpha u_{0} G_{\alpha}+v_{\alpha} H_{0}^{\prime}=0 \\
& H_{0}=\frac{\gamma-1}{\rho_{0} \mu_{\infty}^{2}}+\frac{u_{0}^{2}}{2} \\
& G_{\alpha}=-\left(1 /\left(\frac{1}{\gamma}-1\right) \mu_{\infty}^{2}\right) R_{\alpha} / \rho_{0}^{2}+u_{0} u_{\alpha} \\
& \rho_{0}=\mu_{0}^{2}\left(u_{0}^{2} \mu_{\infty}^{2}\right) \rho_{0}=\rho_{0}^{\prime} v_{1}\left(\alpha u_{0}\right),
\end{aligned}
$$

где

$$
()^{\prime}=\frac{\partial}{\partial y}
$$

Если ввести новую переменную $\mathrm{z}=\frac{\mathrm{u}_{0}}{\mathrm{u}_{0}^{\prime}}$, то можно свести систему (8) к одному дифференциальному уравнению

$$
z^{\prime \prime}+2 z^{\prime}\left(\frac{\mu_{0}^{\prime}}{\mu_{0}}\right)+z\left(-\beta+2\left(\frac{\mu_{0}^{\prime}}{\mu_{0} \alpha^{2}}\right)\right)=0
$$

с граничными условиями

$$
\begin{array}{ll}
\mathrm{V}_{1}(0)=0 ; & \mathrm{V}_{\mathrm{l}}(\infty)=0 ; \\
\mathrm{z}(\infty)=0 ; & \mathrm{z}(0)=\text { const. }
\end{array}
$$

Это уравнение представляет собой задачу на собственные значения. Существует два пути решения уравнения (10) с граничными условиями (11): поиск собственных значений матрицы, получающейся при разностном представлении уравнения (10); второй путь - как результат решения дифференциального уравнения методом Рунте - Кутта.

На рис. 1 и 2 представлены профили скорости z для различных значений $g, \mu_{\infty}, \gamma$. 


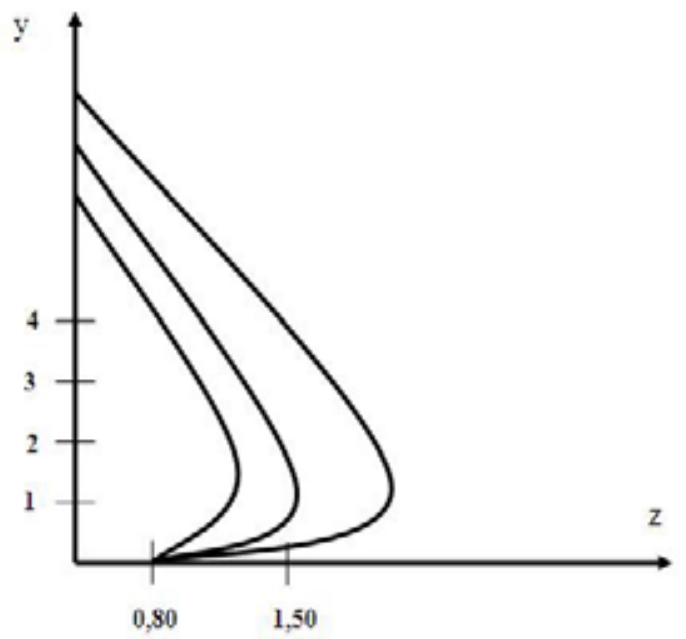

Рис. 1. График распределения скорости z при $\mu=0,2, g=2,5, \gamma=1,8, \alpha=15, \beta=0,01$,

$$
\begin{aligned}
& \mu=0,2, g=0,6, \gamma=1,8, \alpha=15, \beta=0,01, \\
& \mu=0,2, g=2,0, \gamma=1,8, \alpha=3,5, \beta=0,01
\end{aligned}
$$

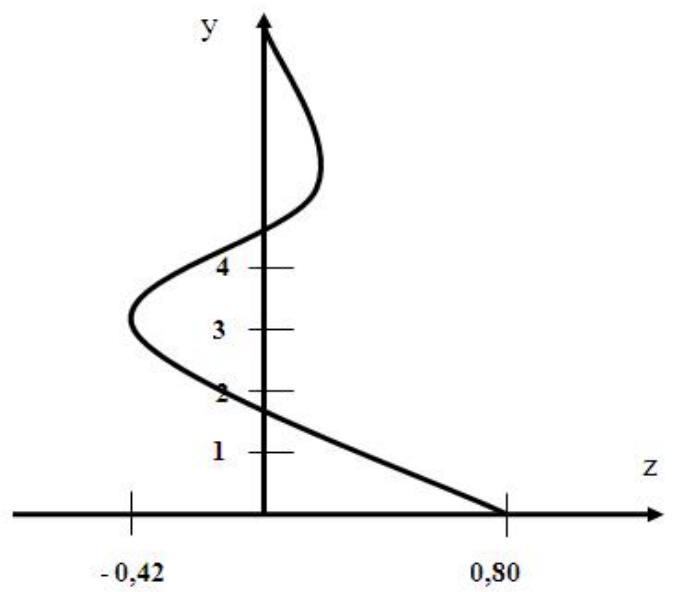

Рис. 2. График распределения скорости z при $\mu=1,4, g=1,4, \gamma=1,8, \alpha=15, \beta=0,01$ 


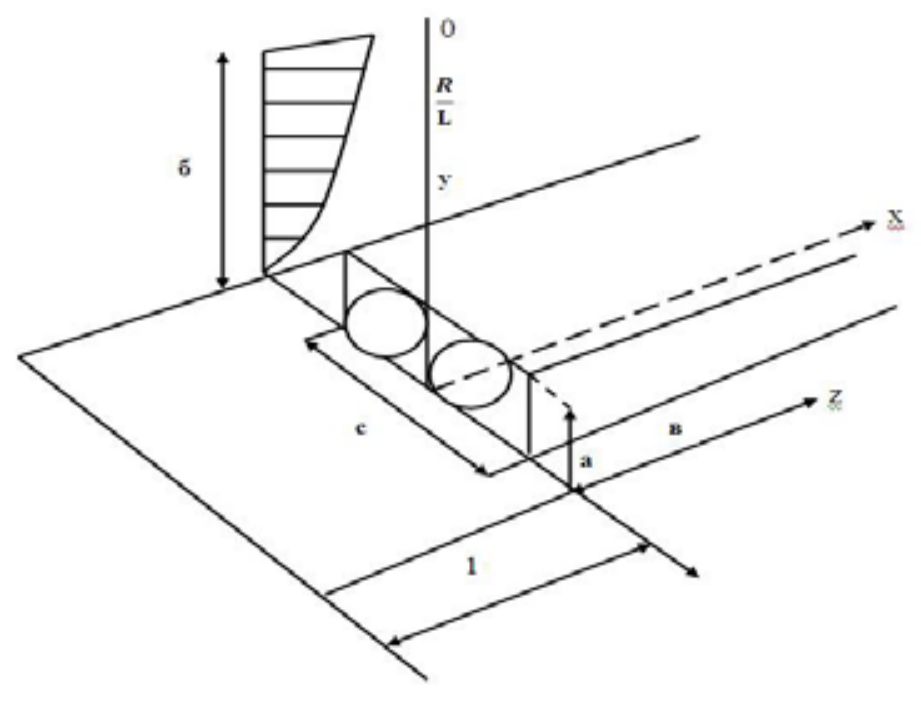

Рис. 3. Схема исследуемого течения

\section{Список литературы}

[1] Ершин Ш.А. Гидроаэродинамика. Алматы, 2013 [Ershin Sh. A. Hydroaerodynamics. Almaty, 2013 (in Russian)]

[2] Ван-Дайк М. Методы возмущений в механике. М.: Мир, 1967 [Van Dyke M. Perturbation methods in mechanics. Moscow, Mir, 1967 (in Russian)]

[3] Peerhossaini H. On the subject of Gortler vortices. Lecture notes in Phisics, ed. S. Zaleski, 1984, 376-384. 